\title{
Medial faces from a concise 3D thinning algorithm
}

\author{
Antoine Manzanera \\ DCE/ETC4/CTA/GIP \\ 16b Av. P. de la Côte d'Or \\ 94114 Arcueil Cedex
}

\author{
ENSTA/LEI \\ 32 Bd Victor \\ 75015 Paris
}

Thierry M.Bernard

\author{
Françoise Prêteux
}

\author{
INT/UP Artemis \\ 9 Rue Charles Fourier \\ 91011 Evry Cedex
}

\section{Bernard Longuet}

\author{
Aérospatiale \\ 2 Rue Béranger \\ 92320 Chatillon Cedex
}

\begin{abstract}
We propose in this paper a new 3D fully parallel thinning algorithm that we believe to be the most concise due to its simple characterization. The algorithm is indeed completely defined by a set of five patterns, three removing conditions and two non-removing conditions. These patterns are designed from the two fundamental and compatible constraints usually expected in skeleta: (1) Topology preservation and (2) Medial surface. From these two constraints, the removing patterns $\left(\alpha_{1}, \alpha_{2}\right.$ and $\left.\alpha_{3}\right)$ detect the non-local maxima, whereas the non-removing patterns $\left(\beta_{1}\right.$ and $\left.\beta_{2}\right)$ prevent any topology change that the removing conditions could imply. We show that the three mentioned constraints are respected. The logical conciseness of our procedure, called $M B-3 D$, makes it to our knowledge the easiest $3 D$ thinning algorithm to implement. Some results are displayed, that illustrate the relevance of our approach.
\end{abstract}

\section{Keywords}

$3 D$ fully parallel thinning algorithm - Discrete topology - Concise Boolean expression.

\section{Introduction}

Skeletonization is a very common way to represent binary shapes with a limited amount of information. A skeleton that faithfully represents a shape is expected to (1) be topologically equivalent to that shape and (2) render its geometry and location. Skeleta are usually obtained through an iterative reduction operator called thinning: certain types of border points are iteratively removed until no more points can be deleted: the remaining image is called the skeleton. Thinning algorithms have been an important subject of research for years in $2 \mathrm{D}$, and more recently in 3D. Lots of efforts have been done to provide the simplest characterization of the non-skeletal points removed by an elementary thinning iteration. In $3 \mathrm{D}$, the characterizations remain complicated, with great number of deleting conditions and exceptions [10], [2], [6], [7], or with special rules to avoid disconnection due to parallel removal [5].

We present in this paper what we believe to be the most computationally efficient to date Boolean expression of a fully parallel 3D thinning process: the nonskeletal points are entirely characterized through a set of three Boolean removing conditions and two Boolean remaining conditions, every condition being defined by a simple pattern, which makes our algorithm straightforward to implement. Our algorithm meets two fundamental (yet compatible) constraints: (1) Topology preservation (2) presence of the local maxima. Constraint (2) ensures that the skeleton is located right at the "middle" of shapes, and renders their most significant geometrical features. The algorithm, called MB-3D, is completely defined by two small families of patterns:

- Patterns $\alpha_{1}, \alpha_{2}$ and $\alpha_{3}$ are designed to remove non local maxima points for the distance induced by the 6 -topology, within the 26-neighborhood.

- Patterns $\beta_{1}$ and $\beta_{2}$ are designed to avoid disconnection of 18 - and 26 -connected points respectively.

For self-containedness purposes, the following section recalls some preliminaries. In Section 3, we present our algorithm, giving the Boolean expression and the visual representation of the patterns. Then we show that with the two simple pattern families that define it, the MB-3D algorithm respects the two constraints stated above. At the same time, we illustrate the paper with some results and discuss the behavior of the algorithm.

\section{Theoretical background}

In this section we set out the mathematical tools necessary to handle the notions we are dealing with. In the first subsection, we present the discrete geometry framework, the cubic grid. Next, we discuss the issue 

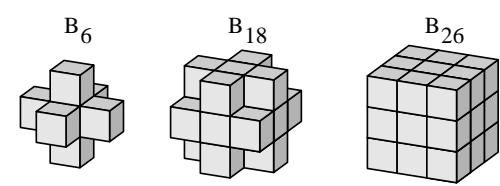

Figure 1: Unity sized balls for the three different topologies in the cubic grid.

of topology preservation, and present the way it has been addressed for the cubic grid in the litterature. Finally, we introduce the morphological operators to be used for the definition of our thinning procedure.

\subsection{Discrete topologies in the cubic grid}

Let $\mathbb{Z}^{3}$ be the discrete space. Let $X \subset \mathbb{Z}^{3}$ a (binary) (three-dimensional) image. Let $X^{c}=\mathbb{Z}^{3} \backslash X$ denote the background of $X$. We are working in the cubic grid, this means that the real space $\mathbb{R}^{3}$ is discretized into $\mathbb{Z}^{3}$ by means of the cubic quantization: A point $z \in \mathbb{Z}^{3}$ represents an elementary volume which is the unit cube centered around $z$. In this mesh, three different connectivity relations can be defined. Figure 1 shows the different topologies in the cubic grid, as defined by the unity sized balls. The topology (and the induced distance) is usually denoted using the number of neighbors in the corresponding type of connectivity. Namely, a point, i.e. a cube in our representation, has 6 (respectively 18,26 ) neighbors in the connectivity defined by $B_{6}$ (respectively $B_{18}, B_{26}$ ) which are the points it shares a face (respectively an edge, a vertex) with. Let $x, y$ be two points of $\mathbb{Z}^{3}$. We say that $x$ is $N$-adjacent to $y(N=6,18$ or 26$)$ if $x$ is a $N$-neighbor of $y$. Let $A, B$ be two subsets of $\mathbb{Z}^{3}$. We say that $A$ is $N$-adjacent to $B$ if there exists $a \in A$ and $b \in B$ such that $a$ is $N$-adjacent to $b$. Let $X \subset \mathbb{Z}^{3}$. $X$ is an $N$-connected component $(\mathrm{N}-\mathrm{cc})$ of $\mathbb{Z}^{3}$ if there does not exist any partition of $X$ into two subsets that are not $N$-adjacent. Let $X \subset \mathbb{Z}^{3}$ be an image. $x \in X$ is said to be $N$-interior to $X$ if all its $N$-neighbors belong to $X$.

Definition 1 Let $d_{N}$ be the distance induced by the $N$-topology. Let $X \subset \mathbb{Z}^{3}$. A ball $\mathcal{B}$ is maximal in $X$ if $\mathcal{B} \subset X$ and there does not exist a ball $\mathcal{B}^{\prime}$ such that $\mathcal{B} \subset \mathcal{B}^{\prime} \subset X$. Let $\mathcal{S}_{N}(X)$ be the collection of the centres of maximum balls associated with $d_{N}$.

Definition 2 Let $X \subset \mathbb{Z}^{3}$. The distance function associated with $d_{N}$ on $X$ is $\Phi_{N}(x)=d_{N}\left(x, X^{c}\right)$.

Property $1 \mathcal{S}_{N}(X)=\{x \in X ; \forall y N$-adjacent to $\left.x, \Phi_{N}(x) \geq \Phi_{N}(y)\right\}$. In other words, the collection of the centres of maximal balls corresponds to the set of local maxima of the distance map.
This formalism aims at giving a sound basis to the notion of medial surface. Indeed, we ensure that the skeleton lies "at the middle" of the shape if we know that it contains the local maxima of the corresponding distance function.

\subsection{Topology preservation}

The topological equivalence is a well known property. A doughnut is equivalent to a coffee cup because they have both exactly one "hole of the same type" (the handle). In 2D and 3D, the topology can be characterized by the so-called fundamental group, i.e a partition of the curves by the homotopic relation (two curves are homotopic if there exists a continuous morphing from one to the other).

To get a sound definition of such topological property in our cubic grid, special care must be taken in the choice of the connectivity. In particular, an object may be crossed by a connected component of the background only if there is a hole through it! In this respect, it is usually chosen the strongest connectivity for the background (i.e. face sharing), and a weaker one for the object itself (i.e. edge or vertex sharing). The connectivity model that is used in this paper is $(26,6)$-connectivity, which means 26-connectivity for the image and 6-connectivity for the background.

Our thinning process works by iterative deletion of sets of points. The central notion around the characterization of the deleted points is simplicity. A point is simple if its deletion does not change the topology. As in $2 \mathrm{D}$, the computation of simplicity can be done within a finite neighborhood of the point. The most concise characterization is provided by Bertrand and Malandain in [1]:

Theorem 1 (Bertrand and Malandain 94)

Let $X \subset \mathbb{Z}^{3}$ be a binary image. Let $x \in X$. Let $X_{26}^{x}$ denote the set of all the 26-neighbors of $x$, except $x$ itself, that belong to $X$, and $\overline{X_{18}^{x}}$ the set of all the 18neighbors of $x$ that do not belong to $X . x$ is simple in $X$ for the (26,6)-connectivity model if and only if the two following conditions hold:

- $x$ is 26-adjacent to only one 26-cc of $X_{26}^{x}$.

- $x$ is 6-adjacent to only one 6-cc of $\overline{X_{18}^{x}}$.

This characterization uses connected components counting only, as in the $2 \mathrm{D}$ case. It is important to notice, however, that simplicity is a property which is strictly individual with respect to a point of the $\mathrm{cu}^{-}$ bic grid. In general, simultaneously removing simple points from a shape leads to topology changes. From this problem arose the notion of simple sets, which are sets of points that can be removed from a shape while 
preserving the topology. Ronse first introduced the concept in [8] for 2D images, concept that was then generalised by Kong in [3] for higher-dimensional images. In these papers, it is shown that a set is simple for the image $X$ if and only if it can be ordered in a sequence of points $\left\{x_{1}, \ldots, x_{n}\right\}$ such that for every $i$ in $\{1, \ldots, n\}, x_{i}$ is individually simple (in the former sense) with respect to $X \backslash\left\{x_{1}, \ldots, x_{i-1}\right\}$. From this property, Ronse proposed in [9] sufficient conditions that were very efficient to prove the soundness of parallel thinning algorithm in 2D. This result has been extended to the $3 \mathrm{D}$ case by $\mathrm{Ma}$ in [4]. We now give Ma's result for the $(26,6)$-connectivity. Let a unit lattice square be the set of four corners of a unit square of the cubic grid, and a unit lattice cube be the set of eight corners of a unit cube of the cubic grid.

Theorem 2 ( $M a$ 94)

Let $X \subset \mathbb{Z}^{3}$ be a binary image. An algorithm that removes points in parallel from a binary $3 D$ shape $X$ preserves (26,6)-connectivity if the two following conditions are satisfied:

- Every subset of $X$ that is contained in a unit lattice square and that is removed by the algorithm is simple.

- No connected component of $X$ contained in a unit lattice cube is completely removed.

This theorem allows to prove the soundness of a parallel thinning algorithm by checking a limited number of configurations.

\subsection{Morphological operators}

We define hereunder the morphological operators needed to provide the Boolean expression of our thinning procedure.

The morphological erosion of an image $X$ by a set $B \subset \mathbb{Z}^{3}$, denoted $X \ominus B$ is the set of all points $x$ of $\mathbb{Z}^{3}$ such that the translated set of $B$ by vector $x$ is completely included in $X$.

The morphological dilation of an image $X$ by a set $B \subset \mathbb{Z}^{3}$, denoted $X \oplus B$ is the set of all points $x$ of $\mathbb{Z}^{3}$ such that the intersection of the translated set of $B$ by vector $x$ with $X$ is non-empty.

A pattern of $\mathbb{Z}^{3}$ is a tuple $(H, M)$ of finite subsets of $\mathbb{Z}^{3}$ such that $H \cap M=\emptyset$.

The Hit-Or-Miss Transform (HMT) of an image $X$ by a pattern $\gamma=(H, M)$ is the image:

$$
X \circledast \gamma=(X \ominus H) \cap\left(X^{c} \ominus M\right) .
$$

We will say that $x$ matches $\gamma$ every time that $x \in$ $X \circledast \gamma$.

If we denote $B_{N}$ the set of all the $N$-neighbors of the origin $(N=6,18$ or 26 , cf. Figure 1$)$, we may also define another transformation that we call Hit-Or-Miss Neighborhood Transform (HMNT) relative to the $N$ neighborhood, that we denote $X \odot_{N} \gamma$, and define by: $X \odot_{N} \gamma=(X \circledast \gamma) \oplus\left(B_{N} \ominus(H \cup M)\right)$

Note that $X \odot_{N} \gamma$ is a superset of $X \circledast \gamma$.

These notions are going to be used in the definition of MB-3D. HMT corresponds to a configuration that the neighborhood of a point must exactly match, whereas HMNT corresponds to a configuration that must be contained in the mentioned neighborhood.

\section{The thinning procedure}

MB-3D is an iterative parallel thinning algorithm, where each iteration deletes from an image $X$ a set of points denoted $m b(X)$, corresponding to certain neighborhood conditions. These conditions are based on patterns that are shown in Table 1. Every pattern actually comes with all its $\pi / 2$ rotated versions around the three axes $O x, O y$, and $O z$.

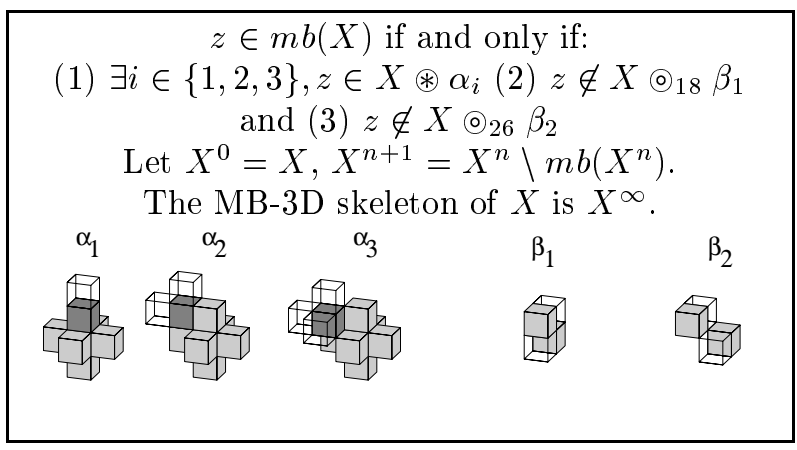

Table 1: Definition of the MB-3D algorithm, based on 5 classes of patterns.

The first collection (the $\alpha_{i}$ family) is used in HMTs. Every pattern represents two subsets of $\mathbb{Z}^{3}$, The Hitset corresponds to the grey cubes, which are the points whose value is 1 . The Miss-set corresponds to the transparent cubes, which are the points whose value is 0 . The dark cube corresponds to the origin. No orientation is given, as every pattern must be considered in all its possible orientations, indeed, the procedure is completely isotropic. Note right away that $\alpha_{1}, \alpha_{2}$ and $\alpha_{3}$ are based on the unity sized ball $B_{6}$ of Figure 1. Thus these patterns naturally lend themselves to computationally efficient description and manipulation. The second collection (the $\beta_{i}$ family) is used in HMNTs, $\beta_{1}$ is to be detected in the 18-neighborhood, $\beta_{2}$ in the 26-neighborhood. Note that no origin is necessary here, since both patterns are symmetrical. To simplify in the following sections we shall say " $x$ matches $\beta_{1}$ " (resp. $\beta_{2}$ ) every time that $x \in X \odot_{18} \beta_{1}$ (resp. $x \in X \odot_{26} \beta_{2}$ ). 


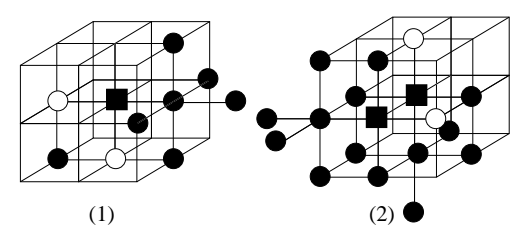

Figure 2: Two examples to illustrate the necessity of patterns $\beta_{i}$.

The thinning action clearly results from the shape of the $\alpha_{i}$ patterns. We actually believe that the definition of these patterns is a very pure characterization of a peeling process: any point that matches an $\alpha_{i}$ is adjacent to a 6-interior point, such that all the faces opposite to this interior point are on the frontier of the image. Still the $\alpha_{i}$ are a bit greedy: some topology changes would occur without the safety provided by the $\beta_{i}$ patterns. Figure 2 shows why the $\beta_{i}$ are necessary through two examples. The black points belong to the image, the white ones to the background. (1) The square point match pattern $\alpha_{1}$, but its removal would lead to 26-disconnection: MB-3D will not remove it since $\beta_{1}$ is contained in its 18 -neighborhood. (2) The two square points match pattern $\alpha_{1}$, but their simultaneous removal would 6-connect the two white points, which is forbidden: MB-3D will not remove them since $\beta_{2}$ is contained in the 26-neighborhood of the black square points.

\section{Results and behavior}

Some results of our thinning algorithm can be seen on Figure 3. The results of MB-3D are displayed on the left column (Images (1.a) to (4.a)). As expected, there are two pixel-thick surfaces. This is a natural outcome of the isotropy constraint.

In this section, we establish the soundness of the procedure, firstly, by proving that the algorithm preserves the $(26,6)$-topology, and secondly by showing that, under a certain condition which is explicited, the skeleton contains the maxima of the $d_{6}$ distances within the 26-neighborhood. We next discuss the behavior of the algorithm as it is applied to some significant shapes.

\subsection{Topological properties}

We prove in this section that the MB-3D algorithm preserves the (26-6)-topology of the binary shapes. If $x \in X$, we use the two sets $X_{26}^{x}$ and $\overline{X_{18}^{x}}$ defined in Theorem 1. The proof is based on five lemmae. Lemmae 1 to 3 deal with the 26-topology preservation of objects, whereas Lemmae 4 and 5 deal with the 6 topology preservation of the background. Lemma 1 and 4 prove that one iteration of the MB-3D algorith$\mathrm{m}$ removes only simple points. Lemma 1 and Lemma 2

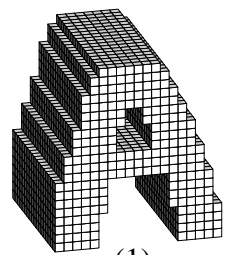

(1)

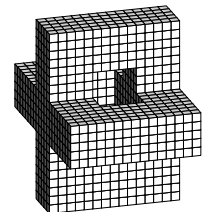

(2)

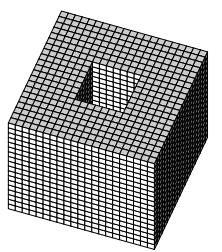

(3)

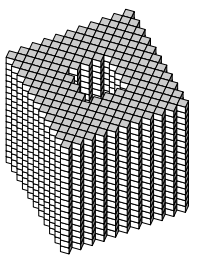

(4)

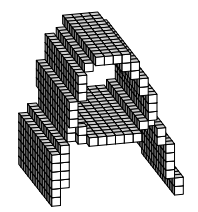

(1.a)

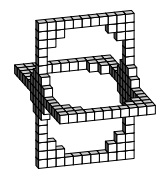

(2.a)

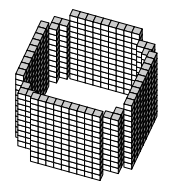

(3.a)

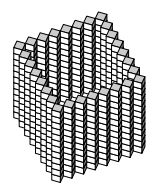

(4.a)
Figure 3: Some results of the thinning algorithm. The left column contains the original images. The right column displays the results of MB-3D.

are used to prove Lemma 3 . Lemma 4 is used to prove Lemma 5. Lemmae 3 and 5 prove that any pair of 6 -adjacent points removed by MB-3D is a simple set. Finally, the proof is completed in Proposition 1.

Lemma 1 Let $x \in X$, between two 6-neighbors a and $b$, with $a \notin X$ and $b \in X$ (cf Figure 6). If $x$ is 26adjacent to more than one 26-cc of $X_{26}^{x}$, then either $x$ is contained in pattern $\beta_{1}$, or $x$ is contained in the pattern $\lambda$ represented on Figure 5.

PROOF

If $x$ is 26 -adjacent to more than one 26cc of $X_{26}^{x}$, then there must exist a point $y$ in $X_{26}^{x}$ which is not 26-adjacent to $b . y$ cannot be a 6-neighbor of $x$, but it may be an 18-neighbor, as illustrated by $c$ on Pattern $\lambda$. Figure 6(1). In that case, since $c$ and $b$ are not in the same 26-cc, $x$ matches $\beta_{1}$. If there is no such $c$, then $y$ is only a 26-neighbor of $x$, as illustrated by $d$ on Figure 6(2). In that case, $x$ matches $\lambda \square$ 

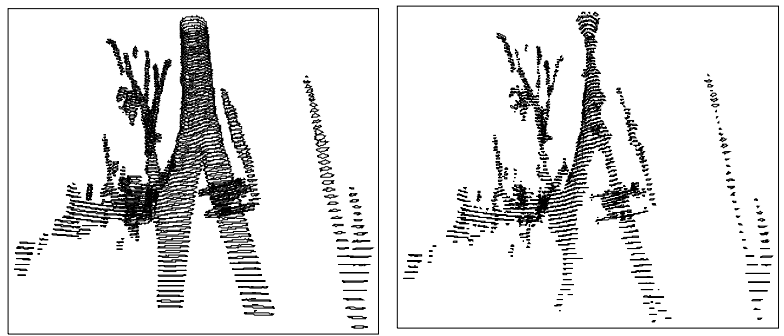

Figure 4: Result of MB-3D on a segmented image of lung.

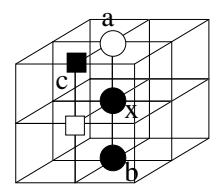

(1)

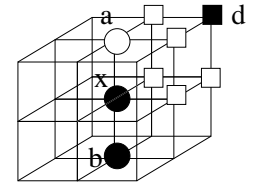

(2)
Figure 6: Proving Lemma 1.

Corollary 1 Any point removed by one iteration of the algorithm fulfils condition 1 of Theorem 1.

Indeed, any point that matches pattern $\alpha_{1}$ or $\alpha_{2}$ is necessarily between two 6 -neighbors, one in $X$, the other in the background. The same holds for a point that matches $\alpha_{3}$, and not $\beta_{1}$. Then Lemma 1 applies and, since pattern $\lambda$ is a particular case of pattern $\beta_{2}$, the point is 26 -adjacent to only one 26-cc of $X_{26}^{x}$.

Lemma 2 Let $x \in X$. Let $Y$ be a subset of $X$ such that $Y \subset m b(X)$ and $Y \cup\{x\}$ is contained in a unit lattice square. Then $x \in(X \backslash Y) \odot_{26} \lambda$ implies $x \in$ $X \odot_{26} \beta_{2}$.

\section{PROOF}

Let us consider $x \in(X \backslash Y) \odot_{26} \lambda$. If $x \in X \odot_{26} \lambda$, then $x \in X \odot_{26} \beta_{2}$. If not, the situation is that of Figure $7(1)$, where $Y \subset\left\{y_{1}, y_{2}, y_{3}\right\}$. Note that the three points represented by squares belong either to $Y$ or to $X^{c}$. If $y_{1} \in X^{c}$ or $y_{3} \in X^{c}$, then obviously $x \in X \odot_{26} \beta_{2}$. If not, $\left\{y_{1}, y_{3}, z\right\} \subset X$. It follows that $y_{2}$ may match an $\alpha_{i}$ only with an interior point within the cube drawn on Figure 7(1). But for each of the seven possibilities, one can easily check that this is not possible. Then $y_{2} \notin Y$, so $y_{2} \in X^{c}$, and the four points $\left\{x, t, y_{2}, z\right\}$ make up a $\beta_{2}$ pattern $\square$

Lemma 3 Let $x$ and $y$ be two 6-neighbors such that $\{x, y\} \subset m b(X)$. Then $x$ is 26-adjacent to only one 26-cc of $(X \backslash\{y\})_{26}^{x}$.

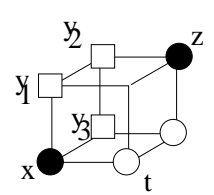

(1)

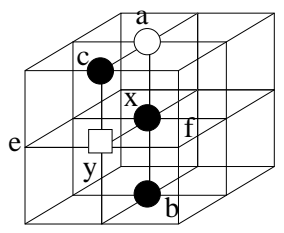

(2)
Figure 7: Proving Lemmae 2 and 3.

PROOF

Under the premises of Lemma 3, it can easily be checked that whatever the $\alpha_{i}$ it matches, $x$ is always between two 6 -neighbors such that one belongs to $X \backslash\{y\}$ and the other to $X^{c}$. Now suppose that $x$ is 26-adjacent to more than one 26-cc of $(X \backslash\{y\})_{26}^{x}$. From Lemma 1, $x$ must match one of the two pattern$\mathrm{s} \beta_{1}$ or $\lambda$ within $(X \backslash\{y\})$. But Lemma 2 shows it cannot be $\lambda$ since $x$ would have matched $\beta_{2}$ before the removal of $y$, in contradiction with $x$ being removed by MB-3D. So $x$ matches $\beta_{1}$ within $(X \backslash\{y\})$; more precisely, the situation of $x$ is that of Figure 6(1), with $c$ and $b$ in distinct 26-ccs. Since $x$ does not match $\beta_{1}$ within $X, y$ as a removed point, is part of $\beta_{1}$, as shown on Figure 7(2). Besides, $e$ and $f$ must both belong to $X^{c}$. But then, $y$ could not have matched an $\alpha_{i}$ pattern, which is in contradiction with its removal by MB-3D $\square$

Lemma 4 Let $x \in X$, between two 6-neighbors a and $b$, with $a \notin X$ and $b \in X$. If $x$ is 6 -adjacent to more than one 6 -cc of $\overline{X_{18}^{x}}$, then $x$ is contained in pattern $\beta_{1}$.

PROOF

See Figure 8(1). If there exists $c \notin X$ such that $a$ and $c$ belong to two distinct 6-ccs of $\overline{X_{18}^{x}}$, then point $d$ such that $d \neq x, d 6$-adjacent to both $a$ and $c$ must belong to $X$. So $x$ matches pattern $\beta_{1}$

Corollary 2 Any point removed by one iteration of the algorithm fulfils condition 2 of Theorem 1.

Lemma 5 Let $x$ and $y$ be two 6-neighbors such that $\{x, y\} \subset m b(X)$. Then $x$ is 6-adjacent to only one 6 - cc of $\overline{(X \backslash\{y\})_{18}^{x}}$.

PROOF

The premises of Lemma 5 (identical to those of Lemma 3 ), implies that $x$ is between two 6 -neighbors such that one belongs to $X \backslash\{y\}$ and the other to $X^{c}$. Now suppose that $x$ is 6 -adjacent to more than one 6-cc of

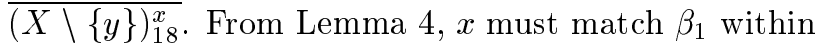




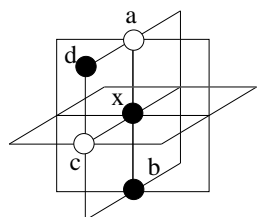

(1)

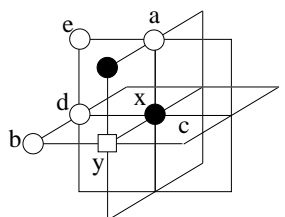

(2)
Figure 8: Proving Lemmae 4 and 5.

$(X \backslash\{y\})$. See Figure 8(2), where $a$ and $y$ belong to distinct 6 -ccs of $\overline{(X \backslash\{y\})_{18}^{x}}$. If $b$ and $c$ both belong to $X$, then $y$ could not have matched an $\alpha_{i}$ pattern, so $b$ or $c$ belong to $X^{c}$. Let us suppose it is $b$. Since $x$ is removed, it does not match pattern $\beta_{2}$, and so $d \in X^{c}$. Since $x$ does not match pattern $\beta_{1}, e \in X^{c}$ also, and finally $a$ and $y$ belong to the same 6 -cc. That leads to a contradiction

We may now give the main proposition.

Proposition 1 The MB-3D algorithm preserves the (26,6) topology.

\section{PROOF}

As mentioned earlier, Lemma 1 and Lemma 4 prove that one iteration of the MB-3D algorithm removes only simple points. Now let $\left\{x_{1}, x_{2}\right\}$ be a pair of 6 adjacent points, simultaneously removed by MB-3D. Lemma 3 and Lemma 5 prove that $\left\{x_{1}, x_{2}\right\}$ is a simple set. More generally, let $Y$ be a set of points such that $Y \subset m b(X)$ and $Y$ is contained in a unit lattice square. Let $x \in Y$ such that $x$ is not simple in $(X \backslash(Y \backslash\{x\}))$. Then Lemmae 1 and 4 , show that $x$ matches pattern $\beta_{1}$ or $\lambda$, but the latter is forbiden by Lemma 2. Then $x$ matches $\beta_{1}$ within $(X \backslash(Y \backslash\{x\}))$. Now let us consider $\left\{x_{1}, x_{2}\right\} \subset m b(X)$ a pair of 18 adjacent, not 6 -adjacent points. It is easy to see that if $x_{1} \notin X \odot_{18} \beta_{1}$, then $x_{1} \notin\left(X \backslash\left\{x_{2}\right\}\right) \odot_{18} \beta_{1}$. So $x_{1}$ is simple in $\left(X \backslash\left\{x_{2}\right\}\right)$, and then $\left\{x_{1}, x_{2}\right\}$ is a simple set. Let $\left\{x_{1}, x_{2}, x_{3}\right\} \subset m b(X)$ be a triplet of points contained in a unit lattice square such that $x_{1}$ and $x_{2}$ are 6 -adjacent. Then $\left\{x_{1}, x_{2}\right\}$ is simple, and it is easy to see that if $x_{3} \notin X \odot_{18} \beta_{1}$, then $x_{3} \notin\left(X \backslash\left\{x_{1}, x_{2}\right\}\right) \odot_{18} \beta_{1}$, so $\left\{x_{1}, x_{2}, x_{3}\right\}$ is a simple set. Let $\left\{x_{1}, x_{2}, x_{3}, x_{4}\right\} \subset m b(X)$ be the four corners of a unit lattice square. $\left\{x_{1}, x_{2}, x_{3}\right\}$ is a simple set, and if $x_{4} \notin X \odot_{18} \beta_{1}$, then $x_{4} \notin\left(X \backslash\left\{x_{1}, x_{2}, x_{3}\right\}\right) \odot_{18} \beta_{1}$, so $\left\{x_{1}, x_{2}, x_{3}, x_{4}\right\}$ is a simple set. Thus we have proved that any set contained within a unit lattice square is a simple set. At last, it is obvious that an iteration of the MB-3D algorithm cannot entirely remove a connected component contained in a unit lattice cube, since no $\alpha_{i}$ fits into this elementary cube. So we have proved that MB-3D is a parallel reduction operator that ful- fils conditions (1) and (2) of Theorem 2. Then MB-3D preserves $(26,6)$-topology $\square$

\subsection{Non-topological properties}

As we have seen in Section 2.1, geometry preservation is related to the notion of medial surface. In the cubic grid, there exist three canonical distances, namely $d_{6}, d_{18}$ and $d_{26}$, leading to three different local maxima sets. A fully parallel thinning algorithm has to favor the 6-distance, since a removed point must be a 6 -contour point (i.e. have a 6 -neighbor in the background). Let $k=6,18$ or 26 . We define the $(6, k)$-medial surface as the following set:

$S_{6}^{k}(X)=\left\{x \in X ; \forall y k\right.$-adjacent to $\left.x, \Phi_{6}(x) \geq \Phi_{6}(y)\right\}$ Note that the case $k=6$ corresponds to the set $S_{6}(X)$ defined in Section 2.1. In order to get a faithful shape representation featuring some noise immunity, the MB-3D algorithm is based on the $(6,26)$-medial surface, i.e. $S_{6}^{26}(X)$.

We illustrate the selective action of the $\alpha_{i}$ by applying the MB-3D to a parallelepiped, firstly restricted to pattern $\alpha_{1}$, secondly to the two patterns $\alpha_{1}$ and $\alpha_{2}$, and finally the complete algorithm. Results can be seen on Figure 9. We see that different skeleta are obtained according to the medial surface they are built on. The skeleton (b) (resp. (c), (d)) is based on the medial surface $S_{6}(X)$ (resp. $S_{6}^{18}(X), S_{6}^{26}(X)$ ). Thus the MB algorithm can lead to different skeleta by the restriction to certain $\alpha_{i}$ patterns. This can be very useful for the versatile representation of complex 3D objects.

As every removed point is adjacen$t$ to a 6-interior point, it can be formally shown that the skeleton contains the set $S_{6}^{26}(X)$ defined above, as long as the points are examined in the order induced by the distance function. This Figure 10: is what appends with usual images. Ill-constructed Nevertheless, there are exceptions, cor- 2D image. responding to ill-constructed images. These images are the 3D equivalent of the better known patholog- (a)

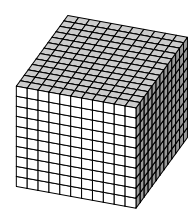

(b)

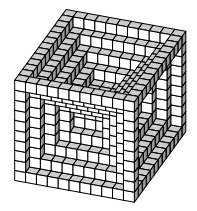

(c)

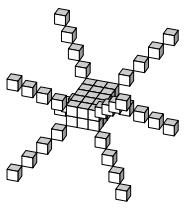

(d)

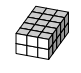

Figure 9: Different choices of the medial surface leading to different skeleta. 


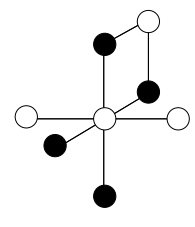

(1)

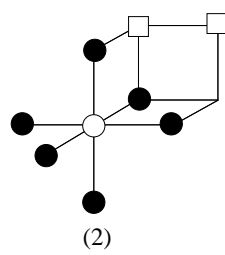

Figure 11: Ill-constructed patterns.

ical images in 2D, of which we give an example on Figure 10. These images correspond to a configuration that would "protect" a piece of surface, preventing a thick volume from being thinned. In $3 \mathrm{D}$, an image is ill-constructed if it contains one of the two patterns shown on Figure 11 (at least one of the two square points does not belong to $X$ ). Note that it corresponds to one-pixel holes matching $\beta_{1}$ or $\beta_{2}$.

The last, but not least, property of MB-3D to be emphasized on is its computational efficiency. Firstly, the conciseness of the Boolean definitions of the procedure leads to a compact computational description, which means efficiency in the computation of one iteration. Secondly, the full parallelism of the algorithm implies that the overall number of iterations needed to achieve the computation of the skeleton equals the radius of the largest 6 -ball contained as many iterations.

\section{Conclusion}

A new thinning algorithm for 3D digital pictures has been proposed. We have given in Table 1 its complete expression. Compared to the other algorithms we know of, MB-3D seems to be the most concise and then the simplest to implement. Indeed, the points removed by the $\alpha_{i}$ patterns are those that are adjacent to a 6-interior point, and for which every face opposite to this interior point is a frontier face. With this very short characterization, the $\alpha_{i}$ patterns allow to obtain the medial surface through a fully parallel and isotropic procedure while preserving connectivity, except in a few cases, taken care of by the even simpler $\beta_{i}$ patterns. Although the definition of the thinning algorithm is much shorter than all other algorithms we are aware of, the results prove to be satisfying.

\section{Acknowledgments}

The authors are extremely grateful to Bertrand Collin and Damien Mercier, from CTA/GIP, for the visualization tool and software support. They wish to thank also the anonymous reviewers for their helpful comments. The volumetric medical image was acquired in the Service de Radiologie Central de l'Hôpital de la Salpetrière (Professor Ph. Grenier).
The lung was segmented by Catalin Fetita from the UP Artemis (INT Evry).

\section{References}

[1] Gilles Bertrand and Grégoire Malandain. A new characterization of three-dimensional simple points. Pattern Recognition Letters, 15:169-175, 1994.

[2] WeiXin Gong and Gilles Bertrand. A simple parallel 3d thinning algorithm. In International Conference on Pattern Recognition, Atlantic City - NJ, pages 188-190, 61990.

[3] T.Y. Kong. On the problem of determining whether a parallel reduction operator for n-dimensional binary images always preserve topology. In SPIE Conference on Vision Geometry - Boston, MA, 1993.

[4] Cherng Min Ma. On topology preservation in 3d thinning. CVGIP: Image Understanding, 59-3:328-339, 1994.

[5] C.Min Ma. A 3d fully parallel thinning algorithm for generating medial faces. Pattern Recognition Letters, 16:83-87, 1995.

[6] C.Min Ma and Milan Sonka. A fully parallel 3d thinning algorithm and its applications. Computer Vision and Image Understanding, 64-3:420-433, 1996.

[7] Kálmán Palágyi and Attila Kuba. A 3d 6-subiteration thinning algorithm for extracting medial lines. Pattern Recognition Letters, 19:613-627, 1998.

[8] Christian Ronse. A topological characterization of thinning. Theoretical Computer Science, 43:31-41, 1986.

[9] Christian Ronse. Minimal test patterns for connectivity preservation in parallel thinning algorithms for binary digital images. Discrete Applied Mathematics, 21:67-79, 1988.

[10] P.K. Saha, B.B. Chaudhuri, and D. Dutta Majumber. A new shape preserving parallel thinning algorithm for 3d digital images. Pattern Recognition, 30-12:1939-1955, 1997. 